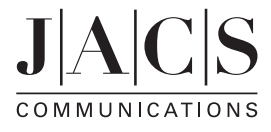

Published on Web 09/21/2004

\title{
Bulk Synthesis of Polypyrrole Nanofibers by a Seeding Approach
}

\author{
Xinyu Zhang and Sanjeev K. Manohar* \\ Alan G. MacDiarmid Laboratory for Technical Innovation, Department of Chemistry, \\ The University of Texas at Dallas, Richardson, Texas 75083-0688
}

Received June 20, 2004; E-mail: sanjeev.manohar@utdallas.edu

We describe a direct, one-step bulk chemical synthetic route to nanofibers of the electronic organic polymer polypyrrole using a variant of our recently described nanofiber seeding method for synthesizing bulk quantities of nanofibers of polyaniline. ${ }^{1}$ This study overcomes a key synthetic challenge in the control of nanostructure of electronic polymers (beyond polyaniline) by uncovering an important chemical property for seed templates to effectively orchestrate fibrillar polymer growth, i.e., the seed template must itself be capable of oxidatively reacting with the monomer (pyrrole, EDOT, etc.).

Polypyrrole is a technologically important, environmentally stable conducting polymer exhibiting high electronic conductivity at physiological $\mathrm{pH}^{2}$ While there are several reports describing the synthesis of polypyrrole fibers within the pores of templates such as zeolites, ${ }^{3}$ alumina,${ }^{4}$ and particle track-etched membranes ${ }^{5}$ or on the surface of nanostructured templates, ${ }^{6}$ the bulk synthesis of nanofibers of polypyrrole directly from pyrrole monomer, i.e., with average fiber diameter $<100 \mathrm{~nm}$, has been a challenge. Approaches such as surfactant-mediated synthesis, ${ }^{7}$ interfacial synthesis, ${ }^{8}$ and nanofiber seeding, ${ }^{1}$ etc., that have been so successful in the synthesis of nanofibers of polyaniline yield only nonfibrous, granular powders in the case of polypyrrole. Fibrillar and tubular morphology in polypyrrole has been observed when large organic dopant anions such as naphthalenesulfonic acid are used during the synthesis. ${ }^{9}$ These fibers and tubes have diameters in the 50-2000 nm range and are formed presumably as a result of the solution aggregation of the dopant anions. In this study we describe a new approach, i.e., the use of reactive seed templates that chemically react with the monomer prior to the addition of oxidant. This prepolymerization reaction on the surface of fibrillar seed templates helps direct the evolution of bulk fibrillar morphology when oxidant is subsequently added. No fibrillar morphology is observed when passive or inert seed templates are used.

The chemical synthesis of polypyrrole nanofibers is very simple and described in greater detail in Supporting Information. It is important to note that unlike the template-directed nanofiber coating of polypyrrole described previously, ${ }^{6}$ only a catalytic amount of seed template is used in this study, e.g., only $1-4 \mathrm{mg}$ of seed template is used for a reaction yield $>300 \mathrm{mg}$.

Granular polypyrrole $\cdot \mathrm{Cl}$ is obtained in unseeded reactions (Figure 1A) or other control reactions seeded by inert seed templates, e.g., $30-50 \mathrm{~nm}$ diameter polypyrrole nanofibers, or 20-30 nm diameter HiPco single-walled carbon nanotube bundles (SWNT) (Figure 1B). Seeding the reaction with 1-4 $\mathrm{mg}$ of $15 \mathrm{~nm}$ diameter nanofibers of $\mathrm{V}_{2} \mathrm{O}_{5},{ }^{10}$ however, dramatically alters the bulk morphology of the product to almost exclusively nanofibers (Figure 1C). The elemental analysis of the nanofibers $(\mathrm{C}, 56.91 ; \mathrm{H}, 3.97 ; \mathrm{N}, 16.53$; $\mathrm{O}, 9.27 ; \mathrm{Cl}, 15.53 ; \mathrm{S}, 0.0 ; \mathrm{V}, 0.0)$ is consistent with the structure $(\mathrm{PPy})(\mathrm{Cl})_{0.37}\left(\mathrm{H}_{2} \mathrm{O}\right)_{0.49}$. These fibers are highly conducting $\left(\sigma_{\mathrm{RT}} \sim\right.$ $50 \mathrm{~S} / \mathrm{cm})$, analytically pure, and free of any $\mathrm{HSO}_{4}{ }^{-}$codopant (normally present in the product when $\mathrm{V}_{2} \mathrm{O}_{5}$ is not used). The

12714 - J. AM. CHEM. SOC. 2004, 126, 12714-12715

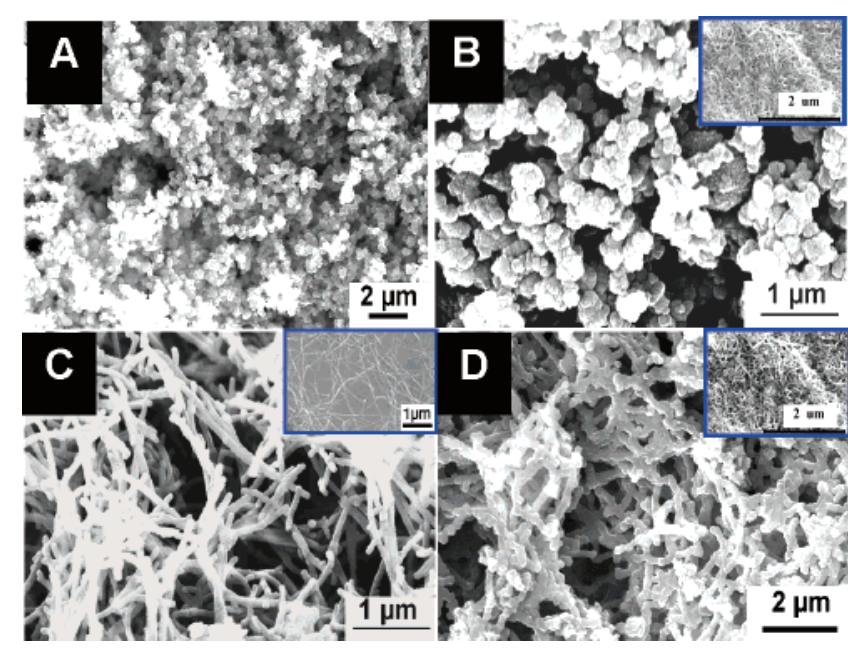

Figure 1. SEM images of polypyrrole $\cdot \mathrm{Cl}$ (insets: seed template): (A) unseeded reaction; (B) seeded with $1.5 \mathrm{mg}$ HiPco SWNT; (C) seeded with $4 \mathrm{mg}$ of $\mathrm{V}_{2} \mathrm{O}_{5}$; (D) seeded with SWNT pre-exposed to $\left(\mathrm{NH}_{4}\right)_{2} \mathrm{~S}_{2} \mathrm{O}_{8}$.

elemental analysis also shows that there is no residual $\mathrm{V}$ in the product, indicating that the $\mathrm{V}_{2} \mathrm{O}_{5}$ seed template is quantitatively removed without the need for additional template removal steps. $\mathrm{V}_{2} \mathrm{O}_{5}$ nanofibers are unstable in aq $1.0 \mathrm{M} \mathrm{HCl}$ and are converted to soluble $\mathrm{V}$ salts during the reaction (see Supporting Information). These results are consistent with two parallel pathways for the $\mathrm{V}_{2} \mathrm{O}_{5}$ seed template, (i) reaction with pyrrole monomer and (ii) dissolution in aq $\mathrm{HCl}$.

The solution darkened noticeably when the $\mathrm{V}_{2} \mathrm{O}_{5}$ seed was added to the pyrrole/ $\mathrm{HCl}$ solution prior to the addition of $\left(\mathrm{NH}_{4}\right)_{2} \mathrm{~S}_{2} \mathrm{O}_{8}$, consistent with the oxidation of pyrrole monomer on the surface of $\mathrm{V}_{2} \mathrm{O}_{5}$. Subsequent addition of $\left(\mathrm{NH}_{4}\right)_{2} \mathrm{~S}_{2} \mathrm{O}_{8}$ results in the rapid precipitation of polypyrrole $\cdot \mathrm{Cl}$ powder having bulk nanoscale morphology. The analogous control reaction seeded with granular $\mathrm{V}_{2} \mathrm{O}_{5}$ also darkened in color, but yielded only granular polypyrrole. In contrast, there was no darkening of the reaction solution when SWNT or other inert seeds were used. Therefore, nanofibrillar morphology is observed in systems in which the seed template must: (i) itself possess nanofibrillar morphology and (ii) also be capable of oxidatively reacting with the monomer.

The need for the seed template to also be chemically reactive toward the monomer is borne out in SWNT-seeded reactions in which the SWNT was pre-exposed to $\left(\mathrm{NH}_{4}\right)_{2} \mathrm{~S}_{2} \mathrm{O}_{8}$ for 20 min before pyrrole monomer was added. In contrast to the "inert" SWNTseeded reaction (Figure 1B), reactions seeded by SWNT preexposed to $\left(\mathrm{NH}_{4}\right)_{2} \mathrm{~S}_{2} \mathrm{O}_{8}$ resulted in polypyrrole $\cdot \mathrm{Cl}$ having nanofibrillar morphology (Figure 1D). It is possible that polymerization is initiated by $\left(\mathrm{NH}_{4}\right)_{2} \mathrm{~S}_{2} \mathrm{O}_{8}$ adsorbed on the SWNT surface in which the nanoscale morphology of the now "active" seed is transcribed across several length scales to the bulk precipitate. Additional evidence for the need for the seed template to be "activated" was 


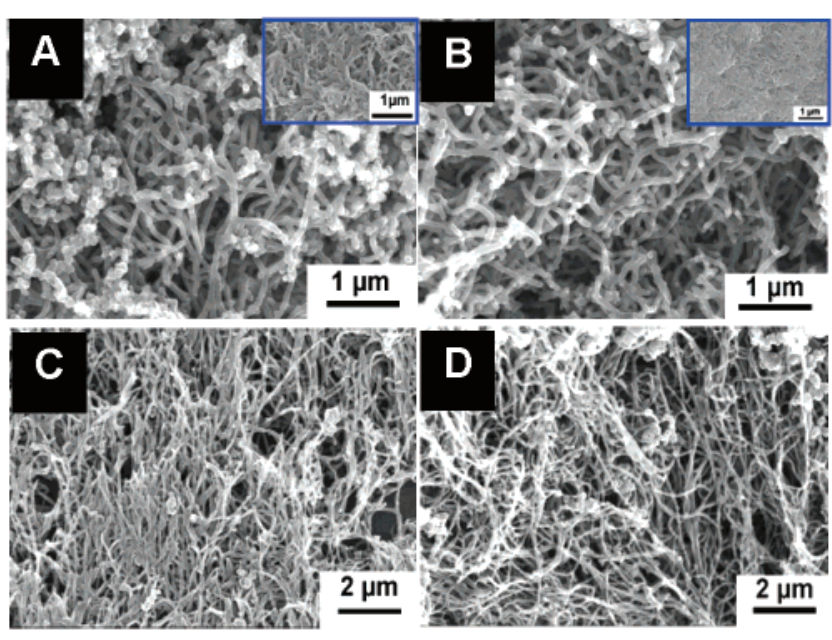

Figure 2. SEM images of polypyrrole $\cdot \mathrm{Cl}$ : (A) bulk powder, seeded by pernigraniline; (B) bulk powder, seeded by aniline dimer; (C) film on glass, seeded by $\mathrm{V}_{2} \mathrm{O}_{5}$; (D) film on poly(ethyleneterephthalate), PET seeded by $\mathrm{V}_{2} \mathrm{O}_{5}$.

obtained in cross-seeding studies using polyaniline as the seed template. For example, granular polypyrrole $\cdot \mathrm{Cl}$ is obtained when the reaction is seeded with polyaniline nanofibers in the emeraldine oxidation state (inert seed), while a significantly larger amount of nanofibrillar polypyrrole $\cdot \mathrm{Cl}$ is obtained when polyaniline nanofibers in the pernigraniline oxidation state (oxidized) is used instead (Figure 2A). The oxidation potential of the pernigraniline oxidation state $\left(V_{\mathrm{oc}}=0.8 \mathrm{~V} \text { vs SCE }\right)^{11}$ is sufficiently high to initiate the oxidation of pyrrole monomer ( $0.5 \mathrm{~V}$ vs SCE). Smooth, uniform polypyrrole $\cdot \mathrm{Cl}$ nanofibers are obtained when the reaction is seeded by nanofibers of the "polyaniline" in the pernigraniline oxidation state synthesized by the chemical oxidative polymerization of the aniline dimer, $\mathrm{N}$-phenyl-1,4-phenylenediamine (Figure 2B).

Normally, there are significant challenges in processing chemically synthesized polypyrrole $\cdot \mathrm{Cl}$ into films, fibers, etc., since it is intractable and insoluble in common organic solvents. However, thin, strongly adherent, and transparent substrate-supported films on glass, plastics, etc., are readily obtained directly from the synthesis without even having to isolate the bulk product (Figure $2 \mathrm{C}, \mathrm{D})$. These films, formed by electroless deposition of polypyrrole on inert surfaces present during the reaction, have been investigated extensively in the past under the umbrella of in situ adsorption polymerization. ${ }^{12}$ The morphology of these films nicely mirrors the bulk powder, permitting the rapid and facile characterization of these nanofibers and in the fabrication of plastic electronic devices and sensors.

Fiber diameter can be controlled when the reaction is carried out in ethanol using $\mathrm{FeCl}_{3}$ as the oxidant and by pre-exposing the $\mathrm{V}_{2} \mathrm{O}_{5}$ seed template to ethanol before the reaction. Thinnest fibers $(30 \mathrm{~nm})$ are obtained by stirring the $\mathrm{V}_{2} \mathrm{O}_{5}$ seed template in ethanol for $30 \mathrm{~min}$, and fiber thickness increases to $100 \mathrm{~nm}$ upon stirring for $12 \mathrm{~h}$ (Figure 3). The reasons for this are not clear, although we believe it could be due to bundling of $\mathrm{V}_{2} \mathrm{O}_{5}$ nanofibers into thicker fibers upon extended stirring in ethanol. It is important to note that polypyrrole fibers obtained with ethanol as the solvent are smoother, more uniform and free of any granular product compared to polypyrrole synthesized under aqueous conditions.

The need to "activate" the seed template to ensure fibrillar polymer growth in polypyrrole vs polyaniline is presumably due to fundamental differences between the two systems. Unlike the polypyrrole system, a small amount of nanofibers are observed even in the unseeded polyaniline system, suggesting that fibrillar polymer
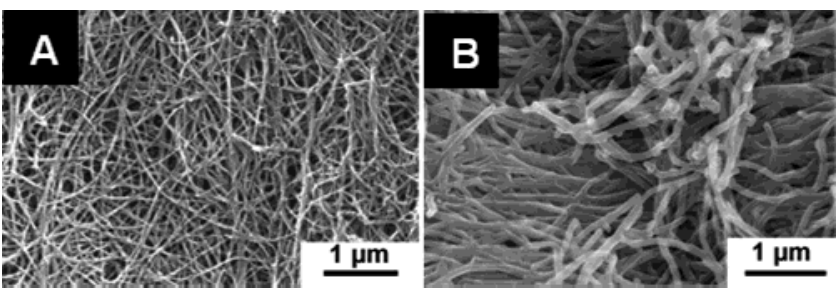

Figure 3. SEM images of polypyrrole $\cdot \mathrm{Cl}$ nanofibers synthesized in ethanol/ $\mathrm{FeCl}_{3}$ using $\mathrm{V}_{2} \mathrm{O}_{5}$ as the seed. Prior to the reaction, $\mathrm{V}_{2} \mathrm{O}_{5}$ was stirred in ethanol for (A) $30 \mathrm{~min}$ and (B) $12 \mathrm{~h}$

growth is intrinsic to polyaniline, and the added seed template directs the synthetic trajectory along these pre-existing pathways. ${ }^{1,8}$ In the polypyrrole system, however, these pathways would have to be induced, e.g., by using either seed templates that are intrinsically reactive toward pyrrole monomer $\left(\mathrm{V}_{2} \mathrm{O}_{5}\right)$ or those that can be rendered reactive by treatment with $\left(\mathrm{NH}_{4}\right)_{2} \mathrm{~S}_{2} \mathrm{O}_{8}$ (SWNT, polyaniline, aniline dimer). It is to be noted that the precise mechanism(s) responsible for the dramatic change in morphology in the presence of nanostructured seed templates (for both systems) remains to be elucidated.

In summary, this study describes for the first time (i) a rapid and convenient seeding method to chemically synthesize bulk quantities of microns-long, 60-90 nm thick nanofibers of polypyrrole, (ii) the use of aqueous acids to quantitatively remove the $\mathrm{V}_{2} \mathrm{O}_{5}$ seed template, which offers a significant advantage over other seed templates (SWNT, polyaniline), (iii) a convenient electroless deposition method to process these nanofibers in the form of thin, strongly adherent coatings on a variety of substrates without any product isolation steps, (iv) control of fiber diameter by using nonaqueous solvents, and (v) a new phenomenon, i.e., the use of reactive seed templates to induce bulk nanoscale morphology. These findings also have potential to be leveraged beyond conducting polymers to embrace the broad class of precipitation polymerization reactions, e.g., they can be used to induce nanoscale morphology in polymerization reactions that are intrinsically recalcitrant to fibrillar polymer growth.

Acknowledgment. We gratefully acknowledge Dr. Alan G. MacDiarmid and Mr. Harsha Kolla for helpful discussions and The University of Texas at Dallas for financial support.

Supporting Information Available: General experimental details (PDF). This material is available free of charge via the Internet at http:// pubs.acs.org.

\section{References}

(1) Zhang, X.; Goux, W. J.; Manohar, S. K. J. Am. Chem. Soc. 2004, 126, 4502 .

(2) Lee, E. S.; Park, J. H.; Wallace, G. G.; Bae, Y. H. Polym. Int. 2004, 53 400 .

(3) Ikegame, M.; Tajima, K.; Aida, T. Angew. Chem., Int. Ed. 2003, 42, 2154

(4) He, J.; Chen, W.; Xu, N.; Li, L.; Li, X.; Xue, G. Appl. Surf. Sci. 2004, $221,87$.

(5) Kumar, S.; Kumar, S.; Chakarvarti, S. K. Phys. Lett. A 2004, 327, 198

(6) Zhang, W.; Wen, X.; Yang, S. Langmuir 2003, 19, 4420

(7) Li, G.; Zhang, Z. Macromolecules 2004, 37, 2683.

(8) Huang, J.; Kaner, R. B. J. Am. Chem. Soc. 2004, 126, 851

(9) Yang, Y.; Liu, J.; Wan, M. Nanotechnology 2002, 13, 771

(10) Bailey, J. K.; Pozarnsky, G. A.; Mecartney, M. L. J. Mater. Res. 1992, 7, 2530.

(11) Manohar, S. K.; MacDiarmid, A. G.; Epstein, A. J. Synth. Met. 1991, 41, 711.

(12) Ayad, M. M. J. Mater. Sci. Lett. 2003, 22, 1577. JA046359V 